ENGEVISTA

Página da revista: http://www.uff.br/engevista/seer/

\title{
Catalisadores multicomponentes com adição de promotores para produção da acronitrila
}

\author{
Laio Damasceno Silva ${ }^{1}$ \\ Luiz Antonio de O. Mascarenhas ${ }^{2}$ \\ Aryane Maria de Oliveira Lima ${ }^{3}$ \\ Icaro Rodrigo Matos Fonseca ${ }^{4}$ \\ Luiz Antônio Magalhães Pontes ${ }^{5}$
}

Resumo: A acrilonitrila é empregada na produção de fibras acrílicas, resinas ABS (acrilonitrila-butadieno-estireno), resinas SAN (poliestireno-acrilonitrila), borracha de acrilonitrila butadieno (NBR), acrilamida, adiponitrila, utilizadas nos setores automobilístico, eletrônico e de eletrodoméstico. O processo mais conhecido para a produção da acrilonitrila é o da amoxidação do propeno. Acetonitrila e ácido cianídrico são coprodutos da reação. Neste trabalho, estudou-se o efeito da substituição do promotor cromo pelo antimônio, visando ao desenvolvimento de um catalisador mais seletivo à acrilonitrila. Foram preparados catalisadores de óxidos metálicos contendo molibdênio, bismuto, ferro, níquel e cério, promovidos com cromo e antimônio. O desempenho catalítico foi medido em um reator de leito fixo a $425^{\circ} \mathrm{C}$, razão molar ar/propeno de 10,5 e amônia/propeno de 1,2. Os produtos foram identificados por cromatografia gasosa e por titulometria. Os catalisadores foram caracterizados por fluorescência de raios $\mathrm{X}$, difração de raios $\mathrm{X}$, termogravimetria e análise textural. A avaliação dos catalisadores na reação de amoxidação do propeno mostrou que o catalisador contendo o antimônio em substituição ao cromo apresentou elevada conversão (61\%) e seletividade (88\%) em acrilonitrila, demonstrando que esta modificação é interessante do ponto de vista do processo.

\footnotetext{
${ }^{1}$ UFBA - Universidade Federal da Bahia

2 UFBA - Universidade Federal da Bahia

${ }^{3}$ UNIFACS - Universidade Salvador

${ }^{4}$ UNIFACS - Universidade Salvador

${ }^{5}$ UFBA - Universidade Federal da Bahia e UNIFACS - Universidade Salvador
} 
ISSN: $1415-7314$

ISSN online: 2317-6717

Palavras-chave: Amoxidação do propeno; acrilonitrila; antimônio; cromo.

\begin{abstract}
Acrylonitrile is employed on the production of acrylic fibers, acrylonitrile-butadiene-styrene (ABS) resins, styrene acrylonitrile (SAN) resins, acrylonitrile rubber, acrylamide, adiponitrile and to obtain many other products used in the automotive, electronics and appliance sectors. The best known process to produce acrylonitrile is the propene ammoxidation. Acetonitrile and hydrogen cyanide are also produced as byproducts in that reaction. Aiming to develop the most acrylonitrile-selective catalyst, the effect of replacing chromium as promoter by antimony was studied in this work. Metal oxide catalysts containing molybdenum, bismuth, iron, nickel and cerium with chromium and antimony as promoters were synthetized. The catalytic performance tests were conducted in a fixed-bed reactor at $425^{\circ} \mathrm{C}$, using an air/propene ratio of 10.5 and an ammonia/propene ratio of 1.2. The products were identified by gas chromatography and titrimetry. The catalysts were characterized by $\mathrm{x}$-ray fluorescence, $\mathrm{x}$-ray diffraction, thermogravimetry and porosity measurements. The results of the catalyst evaluation on the propene amoxidation have shown that the catalyst containing antimony, replacing the chrome, presented higher activity (61\%) and selectivity (88\%) to acrylonitrile, demonstrating that this modification is interesting from the point of view of the process.
\end{abstract}

Keywords: Propene ammoxidation; acrylonitrile; antimony; chrome. 


\section{Introdução}

A acrilonitrila é matéria-prima para a produção de fibras acrílicas, resinas, borrachas sintéticas, fibras de carbono e de uma variedade de outros produtos químicos. Uma das razões da versatilidade da acrilonitrila é a sua capacidade de formar copolímeros com compostos insaturados, tais como a resina ABS (acrilonitrila-butadieno-estireno), a resina SAN (acrilonitrila-estireno) e a borracha nítrilica conhecida como NBR (acrilonitrila-butadieno). Outros produtos que também utilizam acrilonitrila na sua produção são a adiponitrila e a acrilamida.

A produção mundial de acrilonitrila ultrapassa cinco milhões de toneladas por ano, sendo projetado um crescimento anual de 3,7\% até 2018, impulsionado principalmente pela demanda das resinas ABS e SAN (CESPI et al., 2014; ZHENG et al., 2015).

A amoxidação do propeno é um processo oxidativo, no qual uma mistura de amônia, oxigênio e olefina gera nitrila e água. A reação principal da amoxidação, que envolve a formação da acrilonitrila, é dada pela Equação 1.

$$
\mathrm{C}_{3} \mathrm{H}_{6}+\mathrm{NH}_{3}+3 / 2 \mathrm{O}_{2} \rightarrow \mathrm{C}_{3} \mathrm{H}_{3} \mathrm{~N}+3 \mathrm{H}_{2} \mathrm{O} \text { (Eq. 1) }
$$

Os catalisadores utilizados na reação são molibdatos contendo bismuto, ferro, níquel, cobalto e promotores (cromo, magnésio, potássio, césio, fosforo, boro, cério, antimônio e manganês) dispersos em sílica (CESPI et al., 2014).

Na produção de acrilonitrila ocorrem reações que levam à formação de coprodutos como o ácido cianídrico e a acetonitrila. O desenvolvimento de catalisadores tem possibilitado o aumento de rendimento em acrilonitrila, minimizando a formação destes coprodutos. Isso tem sido possível a partir de esforços desenvolvidos na modificação de catalisadores, com uso de promotores ou em melhorias no processo de fabricação. Drenski et al. (1990), Paparizos et al. (1992) e Brazdil et al. (2012) identificaram a presença de diversos metais como agentes promotores de catalisadores usados na obtenção da acrilonitrila. Entretanto, a ação desses promotores ainda não está completamente esclarecida e os trabalhos relatam apenas os efeitos dos promotores que aumentaram a seletividade ao produto principal.

A reação de amoxidação ocorre através do catalisador multicomponente, em que a fase ativa molibdênio-bismuto é regenerada com oxigênio, presente na superfície do catalisador (GRASSELLI, 1999). O cromo e o antimônio vêm sendo utilizados como promotores dos catalisadores de amoxidação para manter o oxigênio na superfície deste sólido; o primeiro metal, em catalisadores de óxidos multimetálicos e o segundo, nos óxidos duplos. Diante disto, a substituição do cromo por antimônio em catalisadores multimetálicos pode ser uma alternativa promissora para aumentar a seletividade em acrilonitrila. 


\section{Experimental}

\subsection{Preparação dos catalisadores}

Foram preparados catalisadores contendo $\mathrm{Mo}, \mathrm{Bi}, \mathrm{Fe}, \mathrm{Ni}, \mathrm{Ce}$ promovidos com $\mathrm{Cr}$ ou $\mathrm{Sb}$, suportados em sílica.

Os precursores dos catalisadores foram preparados através do processo de coprecipitação, pela mistura simultânea de soluções dos nitratos metálicos, heptamolibdato de amônio $\left(\left(\mathrm{NH}_{4}\right)_{6} \mathrm{Mo}_{7} \mathrm{O}_{24} \cdot 4 \mathrm{H}_{2} \mathrm{O}\right)$, solução dos promotores $\left(\mathrm{CrO}_{3}\right.$ ou $\left.\mathrm{Sb}_{2} \mathrm{O}_{3}\right)$ e de uma suspensão em sílica. A metodologia de preparação foi baseada em Paparizos et al., (2005).

O nitrato de ferro, nitrato de níquel, nitrato de bismuto, nitrato de cério foram misturados e aquecidos a $70^{\circ} \mathrm{C}$, até formar uma solução homogênea. Nesta solução, foram adicionados o heptamolibdato de amônio dissolvido em $50 \mathrm{~mL}$ de água destilada, o promotor $\left(\mathrm{CrO}_{3} \operatorname{com~} 0,19 \mathrm{~g}\right.$ ou $\left.\mathrm{Sb}_{2} \mathrm{O}_{3} \operatorname{com} 0,12 \mathrm{~g}\right)$ dissolvido em $5 \mathrm{~mL}$ de água destilada e uma suspensão de sílica (8,34 g). A solução resultante foi aquecida, sob agitação, mantendo-se a temperatura em torno de $100{ }^{\circ} \mathrm{C}$, durante $1 \mathrm{~h}$, seca em estufa a $110{ }^{\circ} \mathrm{C}$ por $16 \mathrm{~h}$ e calcinada. A calcinação foi divida em três etapas com rampa de aquecimento de $10{ }^{\circ} \mathrm{C} / \mathrm{min}$. Na primeira etapa elevou-se a temperatura até $290{ }^{\circ} \mathrm{C}$, mantendo-a por $3 \mathrm{~h}$; na segunda etapa aqueceu-se até $425^{\circ} \mathrm{C}$, permanecendo por mais $3 \mathrm{~h}$; na terceira etapa aqueceu-se então até $570{ }^{\circ} \mathrm{C}$, mantendo-se na temperatura final, também por $3 \mathrm{~h}$.

\subsection{Caracterização dos catalisadores}

Para determinação da composição química dos catalisadores foi utilizada a técnica de fluorescência de raios X (FRX) em um espectrômetro da marca Rigaku modelo RIX 3100. Na realização destas análises, cerca de $500 \mathrm{mg}$ de cada amostra foram primeiramente pastilhados com um diâmetro de 1,8 cm para, então, serem submetidos à análise.

Medidas de difração de raios X (DRX) foram realizadas em um difratômetro da marca XRD6000 Shimadzu, com tubo de cobre. Operou-se com radiação CuK $\alpha(40 \mathrm{KV}, 30 \mathrm{~mA})$, fazendo uso de um monocromador de grafite. A faixa angular estudada foi de $5^{\circ}$ a $80^{\circ}$ com incrementos de $0,05^{\circ}$, utilizandose um tempo de contagem de um segundo por passo.

A análise de termogravimetria (TG) foi realizada numa termobalança da marca Shimadzu, modelo TGA-51. Os ensaios foram executados no intervalo de temperatura de 25 até $1100{ }^{\circ} \mathrm{C}$ com massa de amostras de cerca de $50 \mathrm{mg}$, em cadinho de platina, em atmosfera dinâmica de $\operatorname{Ar}(50 \mathrm{~mL} / \mathrm{min})$ e taxa de aquecimento de $10{ }^{\circ} \mathrm{C} / \mathrm{min}$. 
As propriedades texturais dos catalisadores foram determinadas por fisissorção de N2. As análises foram realizadas num equipamento ASAP modelo 2020 da Micromeritics. A área específica foi obtida utilizando o método de BET (Brunauer, Emmet e Teller) e o volume específico e o diâmetro de poros foram obtidos pelo método $\mathrm{BJH}$ a partir da isoterma de adsorção. As amostras foram primeiramente submetidas a um tratamento térmico de secagem a $200{ }^{\circ} \mathrm{C}$ sob vácuo de $1 \mu \mathrm{mHg}$.

\subsection{Avaliação dos catalisadores}

A reação foi conduzida em um microrreator tubular de vidro com leito fixo, de diâmetro interno $15 \mathrm{~mm}$, operando à pressão atmosférica. Em cada ensaio, utilizou-se uma massa de $2 \mathrm{~g}$ de catalisador. O reator foi aquecido em um forno cilíndrico, circundado por uma resistência elétrica ligada a um programador de temperatura Therma TH 91DP203-000. A temperatura do reator medida por um termopar tipo $\mathrm{K}$ fixado no centro do microrreator, foi de $425{ }^{\circ} \mathrm{C}$. As condições do teste encontram-se descritas na Tabela 1.

As alimentações das matérias-primas, propeno, amônia e ar, foram controladas por medidores mássicos de vazão. A vazão total foi de $4 \mathrm{NL} / \mathrm{h}$, sendo 3,2 NL/h de ar, $0,31 \mathrm{NL} / \mathrm{h}$ de propeno e $0,5 \mathrm{NL} / \mathrm{h}$ de amônia.

Tabela 1. Condições empregadas na avaliação dos catalisadores obtidos.

\begin{tabular}{l|l}
\hline \multicolumn{1}{c|}{ Condições } & \multicolumn{1}{c}{ Valor } \\
\hline Temperatura de reação & $425^{\circ} \mathrm{C}$ \\
\hline Pressão de reação & $1 \mathrm{~atm}$ \\
\hline Razão molar amônia/propeno & 1,2 \\
\hline Razão molar ar/propeno & 10,5 \\
\hline Vazão total dos reagentes & $4 \mathrm{NL} / \mathrm{h}$ \\
\hline $\begin{array}{l}\text { Velocidade especial mássica } \\
\text { (WHSV) }\end{array}$ & $0,31 \mathrm{~h}^{-1}$ \\
\hline Tempo de reação & $30 \mathrm{~min}$ \\
\hline
\end{tabular}

A reação foi ac ompanhada coletando-se amostras dos efluentes do microrreator. A amostra passou através de dois kitassatos (equipados com tubos de dispersão de gases): o primeiro contendo uma solução de ácido clorídrico 0,5 N, para a absorção do ácido cianídrico, e o outro, contendo água para absorção dos orgânicos (acrilonitrila, acetonitrila) e amônia não reagida. Uma ampola, para coleta dos gases não condensáveis (propeno, propano, $\mathrm{O}_{2}, \mathrm{CO}_{2}$ e $\mathrm{CO}$ ), ligava os kitassatos ao totalizador de volume (Wet Test Meter). A solução de ácido clorídrico e a água foram quantitativamente misturadas e analisadas por titulometria e por cromatografia gasosa. A Figura 1 ilustra a unidade de testes catalíticos. 


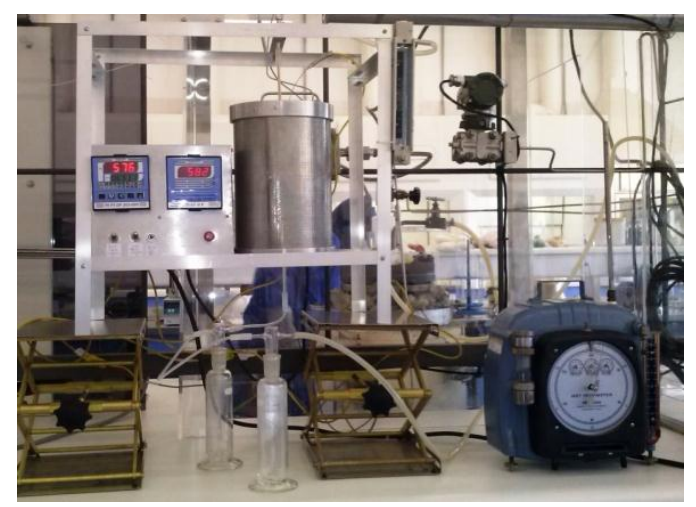

Figura 1. Unidade do teste catalítico usada na avaliação dos catalisadores.

Determinação analítica dos produtos:

O ácido cianídrico foi determinado por titulometria com nitrato de prata, onde o cianeto foi extraído com a adição da solução contendo hidróxido de sódio, hidróxido de amônio e iodeto de potássio. O ácido cianídrico contido na alíquota tomada para análise foi calculado a partir da quantidade de nitrato de prata consumida na reação, conforme a Equação 2. A mesma metodologia foi utilizada por Medeiros (2006).

$$
\mathrm{HCN}=\frac{\left[\left(V_{1}-V_{2}\right) \cdot \mathrm{N} \cdot 0,054 \cdot 10^{6}\right]}{V_{0}}(\text { Eq. 2) }
$$

em que:

V1 = volume de AgNO3 usado para titulação da amostra;

V2 = volume de AgNO3 usado para titulação do branco;

$\mathrm{N}=$ normalidade da solução de $\mathrm{AgNO}_{3}$;

$0,054=$ miliequivalente grama do $\mathrm{HCN} ; 106$ = fator $\mathrm{mg} / \mathrm{L}$;

$\mathrm{V} 0=$ volume da amostra $(\mathrm{mL})$.

A análise dos orgânicos condensáveis (acrilonitrila e acetonitrila) foi realizada em um cromatógrafo a gás HP-5890 com detector de ionização em chama e coluna de vidro, de 3 metros, 1/8”OD, preenchida com Porapak Q, mesh 60/80. A razão de divisão da amostra (split) foi de 1/80. A rampa programada para a corrida cromatográfica foi de $70^{\circ} \mathrm{C}$, por $6,5 \mathrm{~min}$, até $180^{\circ} \mathrm{C}$.

Os gases incondensáveis, não absorvidos (propeno, dióxido de carbono, monóxido de carbono, oxigênio e o nitrogênio) coletados em ampola foram analisados em um cromatógrafo a gás Agilent 6890, com detectores de condutividade térmica, usando hélio como gás de arraste com vazão $1 \mathrm{~mL} / \mathrm{min}$ e colunas recheadas com 35\% DC-200 sobre Chomosorb DAW e 18\% bis adipato sobre chromosorb PAW. A rampa programada para a corrida cromatográfica foi de $90{ }^{\circ} \mathrm{C}$, por $6,0 \mathrm{~min}$, até $200^{\circ} \mathrm{C}$. 


\section{Resultados e discussão}

\subsection{Composição química}

Os resultados da composição química dos catalisadores preparados com diferentes promotores ( $\mathrm{Sb}$ e $\mathrm{Cr}$ ) estão apresentados nas Tabelas 2 e 3 com o desvio (Equação 3) dos catalisadores.

$$
\text { Desvio }(\%)=\frac{(\mathrm{TN}-\mathrm{TE})}{\mathrm{TN}} \times 100(\text { Eq. } 3)
$$

onde o teor nominal (TN) é a composição mássica medida e o teor experimental (TE) é a composição mássica determinado pela análise fluorescência de raios-X.

Tabela 2. Composição nominal e experimental das amostras do catalisador contendo cromo.

\begin{tabular}{c|c|c|c}
\hline \multirow{2}{*}{ Elemento } & \multicolumn{3}{|c}{ Promotor Cr } \\
& TN & TE & $\begin{array}{c}\text { Desvio } \\
(\%)\end{array}$ \\
\hline $\mathrm{Mo}$ & 25,00 & 26,10 & -4 \\
\hline $\mathrm{Bi}$ & 1,00 & 0,95 & 5 \\
\hline $\mathrm{Fe}$ & 2,00 & 1,92 & 3 \\
\hline $\mathrm{Ni}$ & 6,00 & 5,80 & 3 \\
\hline $\mathrm{Ce}$ & 1,00 & 0,97 & 3 \\
\hline $\mathrm{Cr}$ & 2,00 & 1,94 & 2 \\
\hline
\end{tabular}

As diferenças percentuais entre o teores nominal e experimental dos metais nos catalisadores de cromo e antimônio foram abaixo de 5\%, obtendo os valores esperados.

Tabela 3. Composição nominal e experimental das amostras do catalisador contendo antimônio.

\begin{tabular}{c|cc|c}
\hline \multirow{2}{*}{ Elemento } & \multicolumn{3}{|c}{ Promotor Sb } \\
\cline { 2 - 4 } & $\mathrm{TN}$ & $\mathrm{TE}$ & $\begin{array}{c}\text { Desvio } \\
(\%)\end{array}$ \\
\hline $\mathrm{Mo}$ & 25,00 & 25,81 & -3 \\
\hline $\mathrm{Bi}$ & 1,00 & 0,99 & 1 \\
\hline $\mathrm{Fe}$ & 2,00 & 1,94 & 3 \\
\hline $\mathrm{Ni}$ & 6,00 & 5,95 & 1 \\
\hline $\mathrm{Ce}$ & 1,00 & 0,98 & 2 \\
\hline $\mathrm{Sb}$ & 2,00 & 1,97 & 2 \\
\hline
\end{tabular}


Os catalisadores apresentaram um menor teor experimental dos metais oriundo dos nitratos. Uma vez que os nitratos utilizados são higroscópicos, a absorção de água pode ter influenciado na pesagem ocasionando um menor valor no teor dos metais.

A partir dos dados mostrados nas Tabelas 2 e 3, pode-se verificar que o método de preparação do catalisador por coprecipitação empregado neste trabalho mostrou-se eficaz.

\subsection{Difração de raios $X$}

Os difratogramas de raios X (DRX) dos catalisadores preparados são apresentados nas Figuras 2 e 3. A identificação das fases foi realizada por comparação com arquivos de dados JCPDS. As amostras apresentaram uma mistura de fases com trióxido de molibdênio [ $\mathrm{MoO}_{3} / \mathrm{JCPDS}$ 76-1003], molibdato de ferro $\left[\mathrm{Fe}_{2}\left(\mathrm{MoO}_{4}\right)_{3} / \mathrm{JCPDS}\right.$ 85-2287], molibdato de níquel [ $\mathrm{NiMoO}_{4} / \mathrm{JCPDS}$ 86-0362], molibdato de bismuto $\left[\mathrm{Bi}_{2} \mathrm{MoO}_{6} / \mathrm{JCPDS} 76-2388\right]$, molibdato de cério $\left[\mathrm{Ce}_{2}\left(\mathrm{MoO}_{4}\right)_{2}\left(\mathrm{Mo}_{2} \mathrm{O}_{7}\right) / \mathrm{JCPDS} 76-1040\right]$. A fase que apresentou maior intensidade foi o trióxido de molibdênio, em concordância com o trabalho de Wu et al. (2009). No catalisador contendo o promotor cromo, além das fases citadas, foram encontradas a fase cromato de níquel [ $\left.\mathrm{Ni}\left(\mathrm{Cr}_{2} \mathrm{O}_{4}\right) / \mathrm{JCPDS} 88-0109\right]$ e a fase molibdato de cromo $\left[\mathrm{CrMoO}_{3} / \mathrm{JCPDS}\right.$ 85-0778]. No catalisador contendo antimônio foi encontrada a fase oxido de molibdato de antimônio $\left[\mathrm{Sb}_{2}\left(\mathrm{MoO}_{6}\right) / \mathrm{JCPDS} 87-0458\right]$.

Como observado no trabalho de Wu et al. (2009) que estudou o efeito da redução e reoxidação do catalisador multicomponente de molibdato de bismulto sobre o seu desempenho na reação de amoxidação pela técnica de DRX, também foram encontradas diversas fases no catalisador virgem como $\mathrm{MoO}_{3}, \mathrm{Fe}_{2}\left(\mathrm{MoO}_{4}\right), \mathrm{FeMoO}_{4}, \mathrm{Bi}_{2}\left(\mathrm{MoO}_{4}\right), \mathrm{Bi}_{2} \mathrm{MoO}_{6}, \mathrm{CoMoO}_{4}$ e $\mathrm{NiMoO}_{4}$.

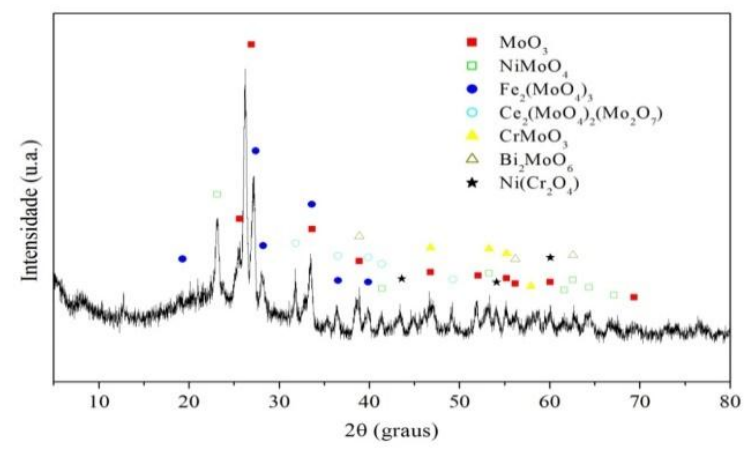

Figura 2. Difratograma do catalisador contendo cromo. 


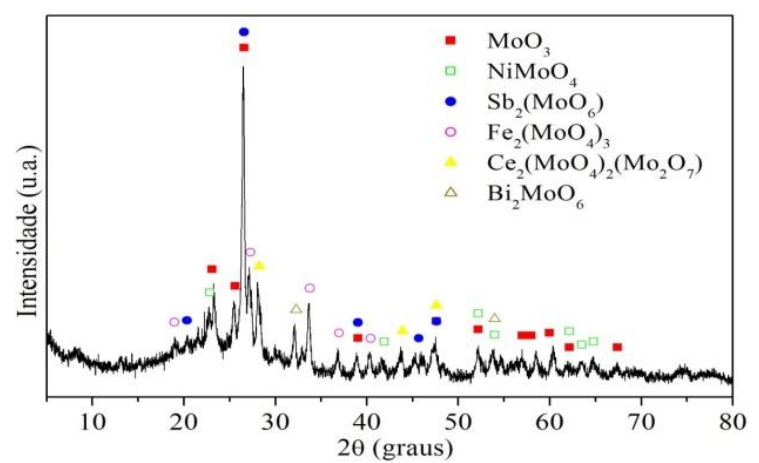

Figura 3. Difratograma do catalisador contendo antimônio.

\subsection{Termogravimetria}

Observando os perfis termogravimétricos dos catalisadores preparados sem a etapa calcinação, verifica-se que o catalisador contendo antimônio (Figura 4), em temperaturas entre 26 e $105{ }^{\circ} \mathrm{C}$, apresentou uma perda de massa de 7,28\%, atribuída à perda de umidade e a materiais voláteis de baixa massa molecular. No intervalo de 105 a $160{ }^{\circ} \mathrm{C}$ houve uma perda insignificante de massa $(1,36 \%)$. Entre o intervalo de 160 a $306^{\circ} \mathrm{C}$ a perda de massa foi de $11,76 \%$, correspondente à saída das hidroxilas para formação dos óxidos. Outra perda de massa insignificante foi na faixa de 306 a $664{ }^{\circ} \mathrm{C}$, com 2,78\%. A maior perda de massa ocorreu a partir da temperatura de $664^{\circ} \mathrm{C}$, evidenciada pelo vale na curva DTG, em $834{ }^{\circ} \mathrm{C}$. Esta perda acentuada de massa é atribuída à sublimação do trióxido de molibdênio. Entre 664 e $1000^{\circ} \mathrm{C}$, houve uma perda de $31,87 \%$. A perda total foi de $55,06 \%$ na faixa de 26 a $1000^{\circ} \mathrm{C}$.

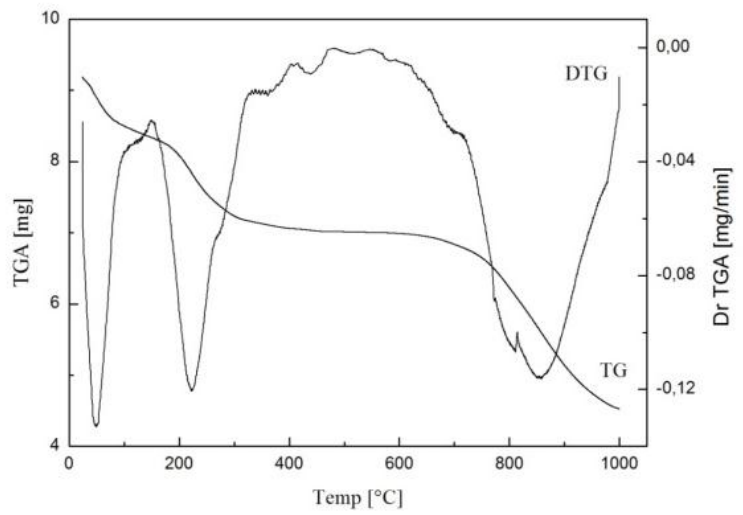

Figura 4. Curvas TG/DTG para as amostras do catalisador contendo antimônio.

O catalisador contendo o cromo (Figura 5) apresentou uma perda de massa de 7,74\% entre a temperatura ambiente e $105^{\circ} \mathrm{C}$, correspondendo à eliminação de umidade e, também, de materiais voláteis de baixa massa molecular. Na faixa entre 105 e $165^{\circ} \mathrm{C}$, a perda de massa foi baixa, com valor de $2,11 \%$, sendo considerada desprezível. A perda de massa de $12,39 \%$ entre 165 e $337^{\circ} \mathrm{C}$ é referente à formação dos óxidos. Na faixa de 337 a $661{ }^{\circ} \mathrm{C}$ não houve uma perda de massa significativa $(2,37 \%)$. Acima de $661^{\circ} \mathrm{C}$, ocorreu uma perda acentuada de massa, evidenciada pelo vale da curva de DTG a 850 
${ }^{\circ} \mathrm{C}$. Entre 661 a $1000^{\circ} \mathrm{C}$, houve uma perda de massa de $26,18 \%$, referente à decomposição do trióxido de molibdênio. A perda de massa total foi de $50,79 \%$ na faixa de 26 a $1000^{\circ} \mathrm{C}$.

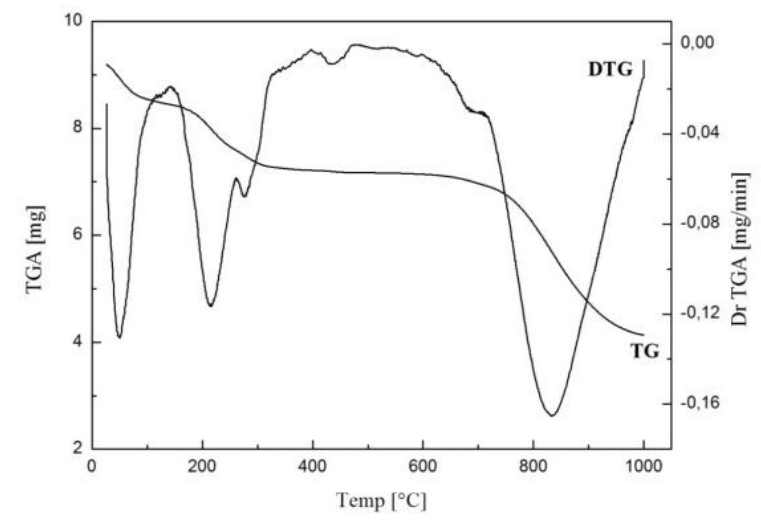

Figura 5. Curvas TG/DTG para as amostras do catalisador contendo cromo.

Os catalisadores preparados tiveram comportamentos similares. Na faixa de 160 a $350{ }^{\circ} \mathrm{C}$, as perdas de massas, como já esclarecido acima, são referentes à formações dos óxidos.

A maior perda de massa observada em ambos os catalisadores, na faixa de 660 a $1000{ }^{\circ} \mathrm{C}$, está relacionada com a sublimação do trióxido de molibdênio. Segundo resultados discutidos por Stecher (1965) e Thang (2005) nos seus trabalhos, a sublimação de trióxidos de molibdênio ocorre na faixa de 700- $1100^{\circ} \mathrm{C}$, corroborando os resultados aqui obtidos.

A temperatura de reação definida foi de $425^{\circ} \mathrm{C}$, no intervalo de 400 a $500{ }^{\circ} \mathrm{C}$ onde não há perda de massa correspondente à fase ativa do catalisador. Apenas impureza retida no cristalito é desprendida.

O catalisador que obteve a formação dos óxidos em temperaturas mais baixas foi o catalisador contendo o antimônio, no intervalo de 165 a $306^{\circ} \mathrm{C}$. Já para o catalisador contendo o cromo, o intervalo foi de 160 a $337^{\circ} \mathrm{C}$. Verifica-se que o catalisador contendo o antimônio apresenta uma vantagem na etapa de calcinação, podendo calcinar com temperaturas mais baixas para formação dos óxidos.

\subsection{Análise textural}

Os dados obtidos para a medida da área superficial e para a porosidade estão apresentados na Tabela 3 para os catalisadores sintetizados contendo o cromo e o antimônio e para o suporte utilizado na preparação dos catalisadores.

Tabela 3. Valores de área superficial específica e porosidade do suporte e dos catalisadores.

\begin{tabular}{c|c|c}
\hline $\begin{array}{c}\text { Suporte e } \\
\text { Catalisadores }\end{array}$ & $\begin{array}{c}\text { SBET } \\
\left(\mathbf{m}^{\mathbf{2}} \mathbf{g}\right)\end{array}$ & $\begin{array}{c}\text { Porosidade } \\
\left(\mathbf{c m}^{\mathbf{3}} / \mathbf{g}\right)\end{array}$ \\
\hline Suporte & 241 & 0,75 \\
Promotor $\mathrm{Cr}$ & 77 & 0,20 \\
\hline
\end{tabular}




\begin{tabular}{l|l|l} 
Promotor Sb & 109 & 0,20 \\
\hline
\end{tabular}

Observa-se, a partir dos dados obtidos pela análise de BET, que a adição dos metais provocou uma redução na área superficial específica do catalisador devido ao recobrimento do suporte.

$\mathrm{O}$ catalisador com o promotor cromo apresentou uma área superficial específica menor do que o catalisador contendo antimônio. Essa diminuição da área superficial, após a impregnação dos metais e calcinação, pode ter sido provocada pela formação de cristais maiores do cromo, identificados pelas análises de DRX dos catalisadores. Verifica-se ainda, pela Tabela 3, que a porosidade para os catalisadores foram $0,2 \mathrm{~cm}^{3} / \mathrm{g}$.

\subsection{Testes catalíticos}

A Tabela 4 mostra as conversões do propeno obtidas com os catalisadores contendo cromo e antimônio. $\mathrm{O}$ catalisador contendo o antimônio apresentou em torno de 10 \% mais atividade que o catalisador com o promotor cromo.

Tabela 4. Comparação entre as conversões dos catalisadores com o promotor cromo e o promotor antimônio.

\begin{tabular}{c|c}
\hline Catalisadores & Conversão $(\mathbf{m o l} \%)$ \\
\hline Promotor $\mathrm{Cr}$ & 50 \\
\hline Promotor $\mathrm{Sb}$ & 55 \\
\hline
\end{tabular}

A Tabela 5 apresenta as seletividades a acrilonitrila dos catalisadores preparados. A seletividade do catalisador contendo antimônio foi um pouco mais alta que a do catalisador contendo cromo.

Tabela 5. Comparação entre as seletividades obtidas dos catalisadores com o promotor cromo e o promotor antimônio.

\begin{tabular}{c|c}
\hline Catalisadores & $\begin{array}{c}\text { Seletividade em } \\
\text { acrilonitrila }(\mathbf{m o l} \%)\end{array}$ \\
\hline Promotor $\mathrm{Cr}$ & 79 \\
\hline Promotor $\mathrm{Sb}$ & 82 \\
\hline
\end{tabular}

Conforme o mecanismo proposto por Grasselli (1999), a amoxidação do propeno ocorre sobre o catalisador multicomponente de molibdato de bismuto em um sistema redox. O par Mo-Bi é reoxidado utilizando o oxigênio dissociado adsorvido no ferro e no promotor. $\mathrm{O}$ ferro desempenha um papel importante no processo de amoxidação, através do par redox $\mathrm{Fe} 3+/ 2+$. O ferro 3+ adsorve e disponibiliza o íon oxigênio para o sítio ativo Bi-Mo. No estado de oxidação 2+, ele é capaz de adsorver 
o oxigênio gasoso, para transformá-lo em íon oxigênio $\left(\mathrm{O}^{2-}\right)$, como pode ser visto nas Equações 4 e 5 (WU et al., 2014).

$$
\begin{gathered}
\mathrm{Fe}^{2+}+1 / 2 \mathrm{O}_{2} \rightarrow \mathrm{Fe}^{3+}+[\mathrm{O}]+\mathrm{e}(\mathrm{E} \mathrm{q} .4) \\
\mathrm{Bi}^{0+}\left(\mathrm{Mo}^{4+}\right)+3 \mathrm{Fe}^{3+} \rightarrow \mathrm{Bi}^{3+}\left(\mathrm{Mo}^{6+}\right)+3 \mathrm{Fe}^{2+} \text { (Eq. 5) }
\end{gathered}
$$

Para obter um alto desempenho com atividade e seletividade mais altas, o catalisador deve manter o equilíbrio entre $\mathrm{Fe}^{3+}$ e $\mathrm{Fe}^{2+}$. Desta forma, promotores tais como o cromo $\left(\mathrm{Cr}^{3+} / \mathrm{Cr}^{2+}\right)$, atuam como um diluente estrutural do íon $\mathrm{Fe}^{3+}$ na matriz bismuto-molibdato (GRASSELLI, 1999). Segundo Castelli (2010), tanto o cromo quanto o antimônio atuam como diluentes estruturais e ajustam o poder de oxidação do sistema. Kajitani et al. (2001), empregaram espectroscopia Mössbauer para estudar o catalisador de óxido duplo (antimônio-molibdênio), verificando que o estado de oxidação do antimônio pode ser 3+ e 5+ (par redox $\mathrm{Sb}^{3+} / \mathrm{Sb}^{5+}$ ). Mori et al. (2006) concluíram que o papel do antimônio é manter o oxigênio disponível na fórmula de $\mathrm{O}^{2-}$ na superfície do catalisador, além de funcionar como par redox. Foi observado um aumento na atividade e seletividade quando o antimônio era adicionado. A estrutura dos antimonatos é composta por espécies com pontes de oxigênio (CARBUCICCHIO et al., 1985) onde o complexo de ligação do metal-oxigênio é mais estável do que uma única espécie correspondente ao elemento (Sb) (BURRINGTON et al., 1983), favorecendo a oxidação do promotor.

$\mathrm{O}$ antimônio na forma de $\mathrm{Sb}^{+5}$ tem número de oxidação mais alto do que o cromo na forma de $\mathrm{Cr}^{+3}$ e é estabilizado em um sistema menos energético, facilitando a

doação de oxigênio para o sistema $\mathrm{Fe}^{3+} / \mathrm{Fe}^{2+}$ que provem o par Bi-Mo de íons $\mathrm{O}^{2-}$,

para a amoxidação (CASTELLI, 2010). Na substituição do cromo por antimônio, o catalisador apresenta funcionalmente e estruturalmente mais estabilidade, mantendo o equilíbrio do ferro. $\mathrm{O}$ antimônio com o par redox $\left(\mathrm{Sb}^{3+} / \mathrm{Sb}^{5+}\right)$ é mais estável que o cromo na forma de $\left(\mathrm{Cr}^{3+} / \mathrm{Cr}^{2+}\right)$.

Distribuição dos produtos da reação de a oxidação:

A Tabela 6 apresenta os valores da concentração dos produtos obtidos sobre os catalisadores na reação de amoxidação do propeno conduzida a $425^{\circ} \mathrm{C}$.

Comparando o catalisador promovido com cromo com aquele promovido com antimônio, verificou-se um pequeno aumento na formação de subprodutos: acetonitrila, ácido cianídrico e monóxido de carbono, em detrimento do produto principal, a acrilonitrila. Como a atividade também foi mais elevada, conclui-se que o antimônio é promissor para substituir o cromo como promotor em catalisadores multimetálicos baseados em bismuto e molibdênio.

Entre os subprodutos, houve maior formação de ácido cianídrico que acetonitrila em ambos catalisadores, o que está de acordo com as observações feitas por Dimian et al. (2008). A Tabela 6 ainda 
mostra que o catalisador contendo antimônio é mais eficiente que o cromo, conduzindo à formação de menor quantidade desses subprodutos.

Tabela 6. Produtos formados sobre catalisador contendo cromo ou contendo o antimônio.

\begin{tabular}{l|c|c|c|c|c|c}
\hline Catalisador & $\begin{array}{c}\text { Acrilonitrila } \\
(\mathbf{m o l} \%)\end{array}$ & $\begin{array}{c}\text { Acetonitrila } \\
(\mathbf{m o l} \%)\end{array}$ & $\begin{array}{c}\text { Ácido } \\
\text { Cianídrico } \\
(\mathbf{m o l} \%)\end{array}$ & $\begin{array}{c}\mathbf{C O}_{2} \\
(\mathbf{m o l} \%)\end{array}$ & $\begin{array}{c}\mathbf{C O} \\
(\mathbf{m o l} \%)\end{array}$ & $\begin{array}{c}\text { Propeno } \\
(\mathbf{m o l \% )})\end{array}$ \\
\hline Promotor $\mathrm{Cr}$ & 39,6 & 2,2 & 4,5 & 2,2 & 1,4 & 50,1 \\
\hline Promotor $\mathrm{Sb}$ & 44,6 & 2,1 & 4,2 & 2,2 & 1,5 & 45,4 \\
\hline
\end{tabular}

\section{Conclusão}

A composição química experimental dos catalisadores ficou bastante próxima da nominal, mostrando que o método de preparo é eficiente para a síntese dos catalisadores. As análises de DRX mostraram diversas fases encontradas no catalisador contendo cromo como promotor. Observou-se este elemento em duas fases: cromato de níquel e molibdato de cromo. Várias fases também foram observadas no catalisador contendo o antimônio como promotor. Entretanto, constatou-se a presença deste apenas na fase de molibdato de antimônio.

No catalisador contendo antimônio, os óxidos foram formados em temperaturas mais baixas. Por outro lado, os óxidos do catalisador contendo cromo necessitaram de temperaturas mais altas para sua formação. Este fato representa uma vantagem do catalisador contendo antimônio em relação àquele contendo cromo, pois na etapa de calcinação pode-se trabalhar em uma faixa de temperatura mais baixa.

O catalisador de Mo-Bi-Fe-Ni-Ce contendo antimônio, em substituição ao cromo, promoveu maior conversão do propeno e apresentou seletividade mais alta a acrilonitrila, devido ao antimônio proporcionar um catalisador mais estável do que o catalisador contendo o cromo, favorecendo a reação de amoxidação.

\section{Referências}

ALLEN, M. D.; POULSTON, S. P.; BITHELL, E. G.; GORINGE, M. J.; BOWKER, M. An XPS, TEM, and TPD Study of the Oxidation and Ammoxidation of Propene Using Mixed Fe-Sb Oxide. Journal of Catalysis. v. 163, p.204-214, 1996.

BRAZDIL, J. F.; TOFT, M. A.; SEELY, M. J.; BESECKER, C. J.; GUSTAFERRO, R. A. Mixed metal oxide ammoxidation catalysts. US 8.153.546, 10 abr. 2012.

BURRINGTON, J. D.; KARTISEK, C. T.; GRASSELLI, R. K. Surface intermediates inselective propylene oxidation and ammoxidation over heterogeneous molybdate and antimonate catalysts. Journal of Catalysis, v. 87, p. 363-380, 1984. 
CARBUCICCHIO, M.; CENTI, G.; TRIFIRÒ, F. The role of excess antimony as a promoter of activity and selectivity in selective oxidation in the Fe-Sb system. Journal of Catalysis, v. 91, p. 85-92, 1985.

CASTELLI, A. New Catalysts for Acrylonitrile. Tesi di dottorato di ricerca in CHIMICA INDUSTRIALE - FACOLTÀ DI CHIMICA INDUSTRIALE - Dipartimento di Chimica Industriale e dei Materiali, ALMA MATER STUDIORUM - Università di Bologna, Itália, 2010.

CESPI, D.; PASSARINI, F.; NERI, E.; VASSURA, I.; CIACCI, L. CAVANI, F. Life Cycle Assessment comparison of two ways for acrylonitrile production: the $S O H I O$ process and an alternative route using propane. Journal of Cleaner Production, v.69, p. 17-25, 2014.

DIMIAN, A. C. e BILDEA, C. S. Chemical Process Design. Weinheim: Wiley-CVH, 2008.

DRENSKI, T. L.; FRIEDRICH, M. S.; PAPARIZOS, C.; SEELY, M. J.; SURESH, D. D. Ammoxidation catalysts containing germanium to produce high yields of acrylonitrile. US 5.770.757, 23 jun. 1990.

GRASSELLI, R. K. Advances and future trends in selective oxidation and ammoxidation catalysis. Catalysis Today, v. 49, p. 141-153, 1999.

HU, Y.; ZHAO, F.; WEI, F.; JIN, Y. Ammoxidation of propylene to acrylonitrile in a bench-scale circulating fluidized bed reactor. Chemical Engineering and Processing: Process Intensification, v. 46, p. 918-923, 2007.

JCPDS - Joint Comission of Powder Diffraction File Sets, International Center for Diffraction Data, Newtown Square, PA.

KAJITANI, Y.; TAKAHASHI, M.; TAKEDA, M. Sb Mössbauer spectra for antimony-molybdenum double oxides. International Journal of Inorganic Materials, v.3, p. 337-340, 2001.

MORI, K.; SASAKI, Y.; MIYAKI, K.; WATANABE, H. Method for producing acrylonitrile, catalyst for use therein and method for preparing the same. US 6723869 B1. 20 abr. 2004.

PAPARIZOS, C.; JEVNE, S. C.; SEELY, M. J. Composições de catalisador e processo de conversão de uma olefina. PI 0316852-2 A, 19 out. 2005.

PAPARIZOS, C.; SHAW, W.G. Catalyst for propylene ammoxidation to acrylonitrile. US 5.134.105, 28 jul. 1992.

STECHER, P. G. Handbook of preparative inorganic chemistry, $2^{\mathrm{a}}$ ed, vol. 2, Academic Press, New York, 1965.

THANG, L. M. Synthesis and Application of Bismuth Molybdates. PhD thesiscin Chemistry Department of Inorganic and Physical Chemistry Universiteit Gent, Bélgica, 2005.

WU, L.; YANG, W.; FRENKEL, A. I. Study of the local structure and oxidation state of iron in complex oxide catalysts for propylene ammoxidation. Catalysis Science \& Technology, v. 4, p. 2512-2519, 2014. WU, X.; YU, G.; CHEN, X.; WANG, Y.; LIU, C. Reduction/reoxidation of a multicomponent molybdate catalyst for propylene ammoxidation. Thermochimica Acta, v. 486, p. 20-26, 2009.

ZHENG, D.; CAI, W.; WANGA, T.; QINA, L.; ZHANGB, Z.; RENB, X.; LIA, J.; YANGA, Z. Pilotscale integrated membrane system for the treatment of acrylonitrile wastewater. Desalination, v. 357, p. 215-224, 2015. 